\title{
EFFECTIVE PRECONDITIONERS FOR LARGE INTEGRAL-EQUATION PROBLEMS
}

\author{
T. Malas ${ }^{1,2}$, Ö. Ergül ${ }^{1,2}$, L. Gürel ${ }^{1,2}$ \\ ${ }^{1}$ Department of Electrical and Electronics Engineering \\ ${ }^{2}$ Computational Electromagnetics Research Center (BiLCEM) \\ Bilkent University, TR-06800, Bilkent, Ankara, Turkey \\ E-mail: tmalas@ee.bilkent.edu.tr, lgurel@bilkent.edu.tr \\ fax: +90-312-2905755
}

Keywords: Preconditioning, integral equation methods, algorithm, large-scale problems.

electromagnetic scattering, multilevel fast multipole

\begin{abstract}
We consider effective preconditioning schemes for the iterative solution of integral-equation methods. For parallel implementations, the sparse approximate inverse or the iterative solution of the near-field system enables fast convergence up to certain problem sizes. However, for very large problems, the near-field matrix itself becomes too crude approximation to the dense system matrix and preconditioners generated from the near-field interactions cannot be effective. Therefore, we propose an approximation strategy to the multilevel fast multipole algorithm (MLFMA) to be used as a preconditioner. Our numerical experiments reveal that this scheme significantly outperforms other preconditioners. With the combined effort of effective preconditioners and an efficiently parallelized MLFMA, we are able to solve targets with tens of millions of unknowns in a few hours.
\end{abstract}

\section{Introduction}

In this paper we consider fast iterative solutions of the integral equation methods, which yield dense linear systems in the form of

$$
\bar{Z} \cdot x=b .
$$

The multilevel fast multipole algorithm (MLFMA) [5] defines a splitting of the $\bar{Z}$ matrix in the form of

$$
\bar{Z}=\overline{\boldsymbol{Z}}^{\mathrm{NF}}+\overline{\boldsymbol{Z}}^{\mathrm{FF}} \text {, }
$$

where $\bar{Z}^{\mathrm{NF}}$ and $\overline{\boldsymbol{Z}}^{\mathrm{FF}}$ corresponds to the near-field and farfield elements, respectively.

In real-life problems, the number of iterations required for convergence rapidly increases as the size of the problem gets larger. Hence, it becomes critical to develop and apply efficient preconditioning techniques for the solution of largescale problems [6].
The sparse approximate inverse (SAI) preconditioner using the near-field matrix pattern is a suitable candidate if the construction of the preconditioner is to be parallelized efficiently [2]. We compare the performance of SAI with respect to the exact solution of the near-field matrix. We show that SAI produces successful results for real-life problems formulated by the combined-field integral equation (CFIE). On the other hand, for the electric-field integral equation (EFIE), which is the only choice for open geometries, SAI does not provide a good approximation to the exact inverse [3]. Since the exact solution of the near-field matrix in infeasible, we iteratively solve the near-field system using SAI as a preconditioner, and this iterative solution is used as the preconditioner of the original matrix equation. We show that 0.1 error tolerance, which can be achieved in a few iterations, suffices for an effective preconditioner. We call this preconditioning scheme NF/SAI.

When an iterative solver is used as a preconditioner as in the case of NF/SAI, the original system must be solved by a flexible solver, such as FGMRES. The difference of FGMRES from GMRES is that FGMRES holds the variable preconditioned Krylov vectors, as well as the unpreconditioned Krylov vectors. Consequently, the memory requirement is doubled. This can be alleviated by solving a system that is closer to the original matrix equation. In fact, MLFMA can also be used for the inner system so that by fixing the inner solver's tolerance to 0.1 , convergence to $10^{-6}$ error can be attained by only six outer iterations. Even though this method provides us a very powerful preconditioner, the total CPU time turns out to be higher with respect to NF/SAI. Since we solve the inner system crudely, such as with 0.1 tolerance, a less accurate but faster MLFMA can help to reduce the CPU time. There can be many ways to decrease the accuracy of MLFMA. Nonetheless, a rigid error-control mechanism, such as decreasing the number of accurate digits, is not optimal. In this paper, we propose a less-error-controlled but very cheap approximation to MLFMA. We carefully reduce the truncation numbers using a tuning parameter, which we call the approximation factor. We propose a strategy to determine the inner tolerance, the maximum allowable inner iterations, and an approximation 
factor so that we optimize the overall solution cost. We call the resulting preconditioner approximate MLFMA (AMLFMA) preconditioner. We show that AMLFMA preconditioner outperforms SAI for both EFIE and CFIE for large-scale problems.

\section{Near-field Preconditioners}

It is customary to construct preconditioners from $\bar{Z}^{\mathrm{NF}}$ assuming it to be a good approximation to $\bar{Z}$. We group these preconditioners as the block-diagonal preconditioner, incomplete factorization methods, SAI, and iterative nearfield schemes.

\subsection{Block-Diagonal Preconditioner}

This is the most widely used preconditioner for CFIE. The block-diagonal preconditioner is usually constructed from the self-interactions of the last-level clusters. Even though it has very low setup time, for complex closed targets, stronger preconditioners has a good potential to improve the convergence rate [11]. For EFIE systems, this preconditioner deteriorates the convergence rate compared to using no preconditioning, hence it should not be used.

\subsection{Sparse Approximate Inverse}

There are various types of SAI preconditioners. Among them, the one that is based on Frobenius norm minimization is successfully used in CEM problems for EFIE [4] and for hybrid-equations [9]. We note that, SAI has a good potential to be helpful for real-life problems formulated by CFIE.

For the SAI preconditioner that depends on Frobenius norm minimization, the sparsity pattern of the approximate inverse should be prescribed. When, the same pattern of $\bar{Z}^{\mathrm{NF}}$ is used for the approximate inverse, significant reduction can be achieved in setup time, because of the block-structure of the near-field matrix [4]. However, filtering may be adequate to gain from memory sometimes [9].

\subsection{Iterative Near-Field Preconditioner}

For ill-conditioned problems such as those produced by EFIE, it is known that SAI is not as successful as ILU when we use the same amount of memory [3]. On the other hand, since SAI is a good approximation to the inverse of the near-field matrix, a fast iterative solution of the system involving nearfield matrix can be obtained and used as a preconditioner.

This approach produces a nested implementation of iterative solvers. In the outer solver that solves the original system, we use FGMRES, a flexible version of GMRES, which allows the preconditioner to change from iteration to iteration. Then, the preconditioner of this solver can be another preconditioned Krylov subspace solver which is called the inner solver. We solve the near-field system in the inner solver, using SAI as the fixed preconditioner. We illustrate this preconditioning scheme in Figure 1.

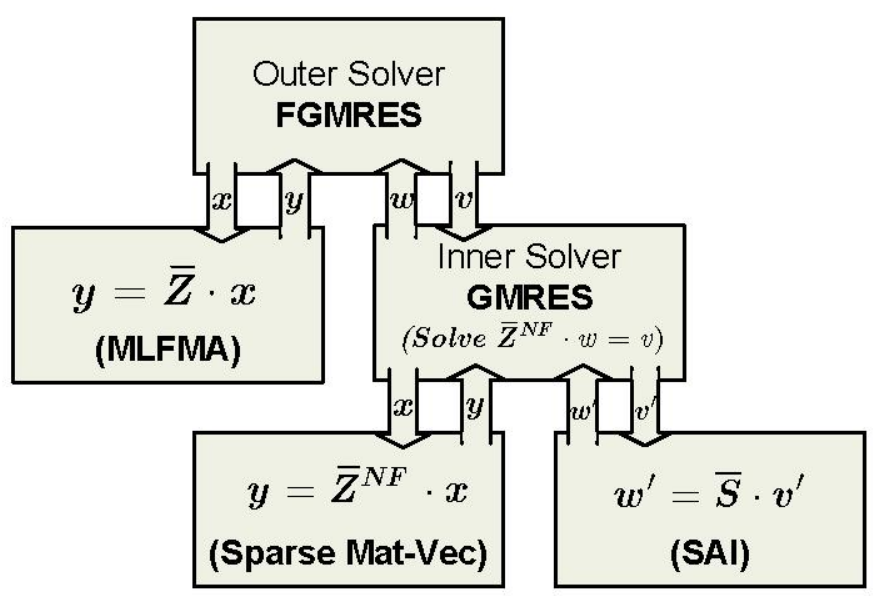

Figure 1: Graphical representation of the iterative near-field preconditioner.

Since the inner solver is used for preconditioning purposes, a rough solution can be adequate. Hence, GMRES is a suitable choice for the inner solver since it provides a fast drop of the residual norm in the early iterations.

\section{Preconditioners Based on MLFMA}

When we have the opportunity to use an iterative procedure for preconditioning as in the iterative near-field scheme, we can also make use of MLFMA to have stronger preconditioners with respect to those obtained from the nearfield matrix. In order to reduce the solution time, cheap versions of MLFMA can be introduced and used for the inner solver. These versions can be obtained by relaxing the accuracy of MLFMA. We achieve an approximate version of MLFMA (AMLFMA) by redefining the truncation number for each level $l$ as

$$
L_{l}^{\prime}=L_{1}+a_{f}\left(L_{l}-L_{1}\right),
$$

where $L_{1}$ is the truncation number defined for the first level, $L_{l}$ is the original truncation number for the level $l$ calculated by using the formula [8]

$$
L \approx 1.73 k a+2.16\left(d_{0}\right)^{2 / 3}(k a)^{1 / 3}
$$

The approximation factor $a_{f}$ is defined in the range from 0.0 to 1.0. As $a_{f}$ increases from 0.0 to 1.0, the AMLFMA becomes more accurate but less efficient, while it corresponds to the full MLFMA when $a_{f}=1.0$ [10].

We fix the stopping criteria of the inner solver at 0.1 and use AMLFMA with $a_{f}=0.2$, which seems a good choice [10]. In general, we set the maximum number of iterations to 10 , to prevent performing unnecessary work when the inner solver stagnates.

\section{Results}

In this section, we demonstrate the performance of the aforementioned preconditioners for EFIE and CFIE formulations. Among the problems that we solved, the square 
patch $(\mathrm{P})$ and the reflector antenna (RA) have open surfaces. Therefore, they are inevitably modeled by EFIE. The closed targets Flamme, which is a stealth geometry [7], and the helicopter $(\mathrm{H})$ are modeled by CFIE. We illustrate these problems in Figure 2 and Figure 3, respectively. More information about the problems is provided in Table 1 and Table 2.

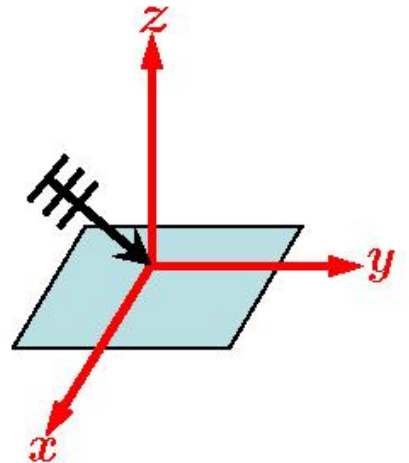

PATCH

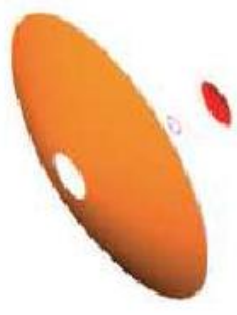

REFLECTOR ANTENNA
Figure 2: Open geometries.

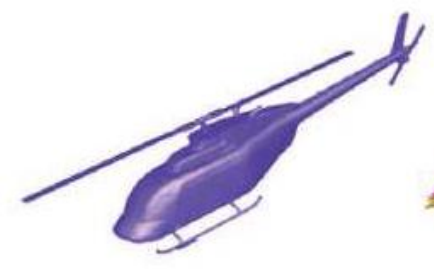

HELICOPTER

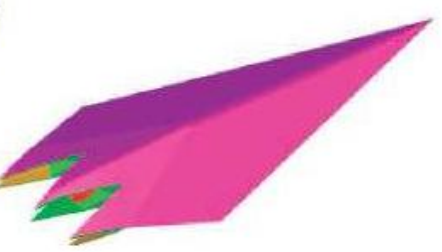

FLAMME
Figure 3: Closed geometries.

\begin{tabular}{|c|r|r|r|}
\hline Problem & $\begin{array}{c}\text { Frequency } \\
(\mathrm{GHz})\end{array}$ & $\begin{array}{c}\text { Size } \\
(\lambda)\end{array}$ & $N$ \\
\hline P1 & 6 & 6 & 12,249 \\
\hline P2 & 20 & 20 & 137,792 \\
\hline P3 & 96 & 96 & $3,164,544$ \\
\hline P4 & 256 & 256 & $21,965,824$ \\
\hline RA1 & 1 & 25 & 356,439 \\
\hline RA2 & 3.73 & 94 & $2,515,103$ \\
\hline
\end{tabular}

Table 1: Information about the open geometries.

\begin{tabular}{|c|r|r|r|}
\hline Problem & $\begin{array}{c}\text { Frequency } \\
(\mathrm{GHz})\end{array}$ & $\begin{array}{c}\text { Size } \\
(\lambda)\end{array}$ & $\boldsymbol{N}$ \\
\hline $\mathrm{F} 1$ & 15 & 30 & 197,892 \\
\hline $\mathrm{F} 2$ & 60 & 120 & $3,581,628$ \\
\hline $\mathrm{H} 1$ & 0.6 & 5 & 185,532 \\
\hline $\mathrm{H} 2$ & 2.76 & 16 & $2,957,61$ \\
\hline
\end{tabular}

Table 2: Information about the closed geometries.

In our experiments we use the GMRES solver for its robustness. We try to reduce the norm of the initial residual by $10^{-6}$ in 1,000 iterations, unless stated otherwise. We perform the parallel tests on a cluster connected via Infiniband network. The nodes have dual XEON 5355 processors and $16 \mathrm{~GB}$ of RAM.

First, we compare SAI and iterative near-field (NF/SAI) preconditioners. We also give the number of iterations for the exact solution of the near-field system (NF-LU) for benchmarking. For SAI, we use the same sparsity pattern of the near-field matrix. For NF/SAI, the stopping criteria of the inner solver is set to one order residual drop or a maximum of 5 iterations. The results presented in Table 3 reveals that such a crude solution of the near-field system outperforms SAI and produce iteration counts that are very close to those of NFLU. The solution times are also significantly reduced compared to SAI.

\begin{tabular}{|c|r|r|r|r|r|r|}
\hline \multirow{2}{*}{$\begin{array}{c}\text { Geo- } \\
\text { metry }\end{array}$} & NF-LU & \multicolumn{3}{|c|}{ SAI } & \multicolumn{2}{|c|}{ NF/SAI } \\
\cline { 2 - 7 } & Iter & Setup & Iter & Soln & Iter & Soln \\
\hline P1 & 26 & 4 & 44 & 12 & 29 & 9 \\
\hline P2 & 53 & 52 & 91 & 336 & 59 & 253 \\
\hline P3 & - & 275 & 253 & 7,6217 & 165 & 5,387 \\
\hline RA1 & - & 952 & 125 & 878 & 71 & 646 \\
\hline
\end{tabular}

Table 3: Comparison of SAI and the iterative near-field preconditioners.

Then, we compare SAI and AMLFMA preconditioners in Table 4. We note that, HS4 and RA2 cannot be solved with SAI or NF/SAI preconditioners. For SP5, the problem is so large that, for SAI preconditioner the available RAM cannot afford the accumulation of the residual vectors of no-restart GMRES. All of these problems can be solved in modest times thanks to the AMLFMA preconditioner. We also note that, for the problems that are solvable by $\mathrm{SAI}$ or NF/SAI, the AMLFMA preconditioner reduces the solution time drastically, as in the SP4 case.

\begin{tabular}{|c|c|c|r|r|c|}
\hline \multirow{2}{*}{$\begin{array}{c}\text { Geo- } \\
\text { metry }\end{array}$} & \multicolumn{2}{|c|}{ SAI } & \multicolumn{3}{c|}{ AMLFMA } \\
\cline { 2 - 6 } & Iter & Soln & Iter & Inner & Soln \\
\hline P3 & 275 & 33,557 & 53 & 526 & 16,184 \\
\hline P4 & - & - & 9 & 85 & 24,689 \\
\hline RA2 & $>1 ; 000$ & - & 322 & 3,205 & 25,740 \\
\hline
\end{tabular}

Table 4: Comparison of SAI and AMLFMA preconditioners. The solution of $\mathrm{P} 4$ is obtained using $10^{-3}$ iterative residual error.

For the closed geometries to be modeled by CFIE, the blockdiagonal preconditioner is commonly used, because of its ease of parallelization and the low setup time. On the other hand, particularly for complex targets such as Flamme and helicopter, we observe that the solution times can be significantly improved by using better preconditioners such as ILU(0), SAI or the AMLFMA preconditioner. We support this claim by comparing the solution of the closed problems in Figure 4 and Figure 5 . For smaller problems that can be solved sequentially, $\operatorname{ILU}(0)$ is a good candidate because of its low setup time [11]. We see that total solution time with $\mathrm{ILU}(0)$ is decreased by more than $50 \%$ for both $\mathrm{F} 1$ and $\mathrm{H} 1$. 
Considering larger problems that can be solved in parallel, we see that for Flamme, even though the iteration counts of SAI are much smaller than those of the block-diagonal preconditioner, because of the larger setup time of SAI there is not a significant difference between the total solution times of these preconditioner. However, for helicopter the gain is around $40 \%$ with SAI. Furthermore, for bistatic RCS calculations, the gains can be much higher. On the other hand, the AMLFMA preconditioner performs outstandingly better with respect to the block-diagonal preconditioner. The solution times is reduced by $35 \%$ for Flamme and $75 \%$ for the helicopter. Hence, for large-scale problems it is wise to construct preconditioners that make use more than the nearfield in an efficient manner.

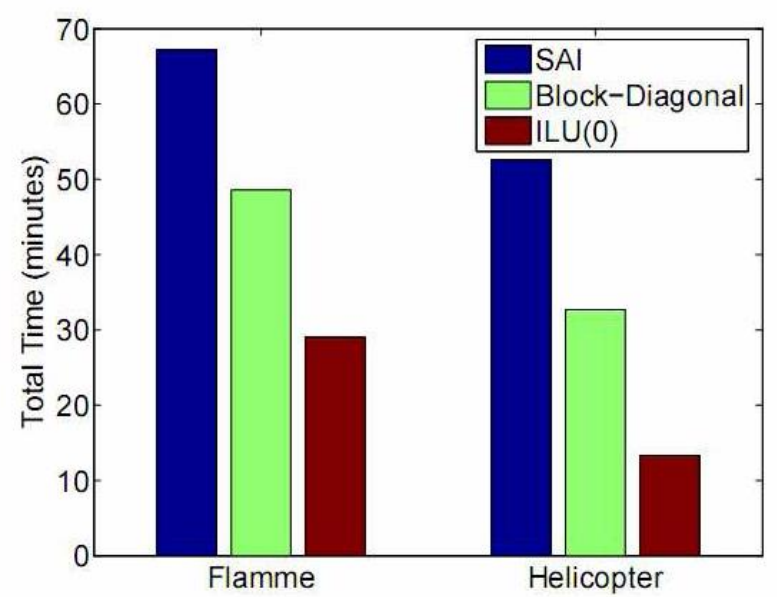

Figure 4: Total solution times (setup + iterations) for F1 and H1.

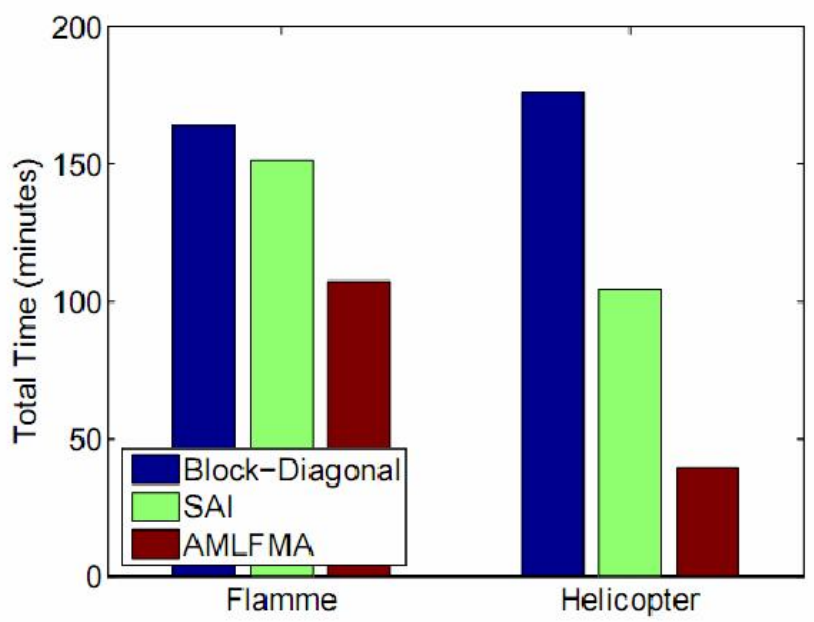

Figure 5: Total solution times (setup + iterations) for F2 and $\mathrm{H} 2$.

\section{Conclusion}

In this work we show that the effectiveness of the integral equation methods can be significantly improved by preconditioning. Even though CFIE is known to produce well-conditioned systems, the solution of the large real-life problems in short times necessitates preconditioning. On the other hand, severely ill-conditioned EFIE systems may not converge without effective preconditioning.

Up to certain sizes, SAI enables fast convergence for both CFIE and EFIE. For EFIE, SAI can be made even stronger by embedding it in an inner-outer solution scheme. On the other hand, for very large problems, the near-field system do not provide a good approximation to dense system matrix. Therefore, preconditioners that are built from the near-field interactions cannot be effective. Considering this fact, we develop the AMLFMA preconditioner. Taking into account the far-field interactions as well as near-field interactions, AMLFMA preconditioner succeeds to solve ultra large EFIE and CFIE systems in reasonable solution times. Our experimental studies reveal the following outcomes for the solutions of large-scale problems using the AMLFMA preconditioner:

- Very large EFIE systems, which are insolvable with other preconditioners, are rendered solvable in moderate solution times.

- Solution times of both EFIE and CFIE problems are decreased by as much as fourfold compared to SAI.

\section{Acknowledgements}

This work was supported by the Scientific and Technical Research Council of Turkey (TUBITAK) under Research Grant 105E172, by the Turkish Academy of Sciences in the framework of the Young Scientist Award Program (LG/TUBA-GEBIP/2002-1-12), and by contracts from ASELSAN and SSM.

\section{References}

[1] S. Balay, W. D. Gropp, L. C. McInnes, and B. F. Smith, "PETSc users manual," Argonne National Laboratory, Tech. Rep. ANL-95/11- Revision 2.1.5, 2004.

[2] M. Benzi, "Preconditioning techniques for large linear systems: a survey," J. Comput. Phys., vol. 182, no. 2, pp. 418-477, 2002.

[3] M. Benzi and M. Tuma, "A comparative study of sparse approximate inverse preconditioners," Applied Numerical Mathematics: Transactions of IMACS, vol. 30, no. 2-3, pp. 305-340, 1999.

[4] B. Carpentieri, I. S. Duff, L. Giraud, and G. Sylvand, "Combining fast multipole techniques and an approximate inverse preconditioner for large electromagnetism calculations," SIAM J. Sci. Comput., vol. 27, no. 3, pp. 774$792,2005$.

[5] W. C. Chew, J.-M. Jin, E. Michielssen, and J. Song, Eds., Fast and Efficient Algorithms in Computational Electromagnetics. Norwood, MA, USA: Artech House, Inc., 2001. 
[6] Ö . Ergül and L. Gürel, "Efficient parallelization of multilevel fast multipole algorithm," in Proc. European Conference on Antennas and Propagation (EuCAP), no. 350094,2006

[7] L. Gürel, H. Bağc1, J.-C. Castelli, A. Cheraly, and F. Tardivel, "Validation through comparison: Measurement and calculation of the bistatic radar cross section of a stealth target," Radio Science, vol. 38, no. 3, pp. 1046-1058, 2003.

[8] M. L. Hastriter, S. Ohnuki, and W. C. Chew, "Error control of the translation operator in 3D MLFMA," Microwave Opt. Technol. Lett., vol. 37, no. 3, pp. 184-188, 2003.
[9] J. Lee, J. Zhang, and C.-C. Lu, "Sparse inverse preconditioning of multilevel fast multipole algorithm for hybrid integral equations in electromagnetics," IEEE Trans. Antennas and Propagation, vol. 52, no. 9, pp. 158-175, 2004.

[10] T. Malas and L. Gürel "The solution of large EFIE problems via preconditioned multilevel fast multipole algorithm", in Proc. European Conference on Antennas and Propagation (EuCAP), 2007.

[11] T. Malas and L. Gürel, "Incomplete LU preconditioning with the multilevel fast multipole algorithm for electromagnetic scattering," SIAM J. Sci. Comput, vol. 29, no. 4, pp. 1476-1494, 2007. 